\title{
Reduction of ferric hemoglobin from Trematomus bernacchii in a partial bis-histidyl state produces a deoxy coordination even when encapsulated into the crystal phase
}

\author{
Antonello Merlino $^{\mathrm{a}}$, Cinzia Verde ${ }^{\mathrm{b}}$, Guido di Prisco ${ }^{\mathrm{b}}$, Lelio Mazzarella ${ }^{\mathrm{a}, \mathrm{c}, \mathrm{d}}$ \\ and Alessandro Vergara ${ }^{\mathrm{a}, \mathrm{c}, \mathrm{d}, *}$ \\ a Department of Chemistry, University of Naples "Federico II", Complesso Universitario \\ Monte S. Angelo, Naples, Italy \\ ${ }^{\mathrm{b}}$ Institute of Protein Biochemistry, CNR, Naples, Italy \\ ${ }^{\mathrm{c}}$ Instituto di Biostrutture e Bioimmagini, CNR, Naples, Italy \\ ${ }^{\mathrm{d}}$ Consorzio Bioteknet, University of Naples, Naples, Italy
}

\begin{abstract}
Crystallographic and spectroscopic evidences on Antarctic fish hemoglobins (AFHbs) have revealed that their ferric tetramers at physiological $\mathrm{pH}$ are in a mixed $\alpha$ (aquo-met) $/ \beta$ (bis-histidyl) coordination state and show a quaternary structure intermediate between the classical $\mathrm{R}$ and $\mathrm{T}$ states (H state). Ferric bis-histidyl adducts (hemichromes) have been also observed in some mammalian Hbs. In order to clarify whether hemichrome in AFHbs can be converted into a ferrous bis-histidyl adduct (hemochrome), at least in the crystal phase, chemical reduction of ferric hemoglobins from Trematomus bernacchii $(\mathrm{HbTb})$ single crystals has been followed via Raman microscopy. The results of this analysis reveal that in HbTb, upon reduction, the bis-histidyl coordination is disrupted in favor of a penta-coordinated ferrous deoxy state, with no evidence of hemochrome. These data are in agreement with UV/Vis absorption spectra in solution. Furthermore, our data are also indirectly supported by the observation that upon reduction with dithionite, the ferric HbTb crystals crack and lose their diffraction power: in the crystalline state, the quaternary structure transition from the $\mathrm{H}$ to the $\mathrm{T}$ state is not compatible with the crystal packing. Altogether these data indicate that if bis-histidyl adducts have a functional significance in AFHbs, this function refers to a stable ferric state, or to a transient, though never detected, ferrous species.
\end{abstract}

Keywords: Roman crystallography, hemicrome, Antarctic fish, hemoglobins

\section{Abbreviations}

$\mathrm{AFHb}$, Antarctic fish hemoglobin;

$\mathrm{Hb}$, hemoglobin;

$\mathrm{HbA}$, human adult hemoglobin;

* Corresponding author: Dr. Alessandro Vergara, Dept. Chemistry, University of Naples, Via Cinthia, 80126 Naples, Italy. Tel.: +39081 674259; Fax: +39081 674090; E-mail: alessandro.vergara@unina.it. 
$\mathrm{HbTb}$, major hemoglobin of Trematomus bernacchii; ox-HbTb, ferric hemoglobin of Trematomus bernacchii at $\mathrm{pH}$ 7.6;

$\mathrm{RR}$, resonance Raman;

$6 \mathrm{cHS}$, hexa-coordinate high spin;

6cLS, hexa-coordinate low spin;

$5 \mathrm{cHS}$, penta-coordinate high spin.

\section{Introduction}

Tetrameric hemoglobins (Hbs) deserve a special position in the field of protein allostery [1-5]. The pioneering achievements of Perutz led to the identification of two distinct $\mathrm{Hb}$ structures: $\mathrm{T}$ (tense) and $\mathrm{R}$ (relaxed) [6]. More recent crystallographic analyses have clearly shown that tetrameric Hbs possess a larger repertoire of structural states [7-14]. Many of these observations are based on studies of Antarctic fish $\mathrm{Hb}(\mathrm{AFHb})$.

Cold-adapted proteins generally differ from their mesophilic or thermophilic homologues only for limited structural characteristics and/or relatively few amino-acid substitutions in functional regions, which can lead to enhanced structural flexibility. Thus, a systematic study of these molecules may facilitate the identification and characterization of accessible conformational substates that result in a better understanding of their functional properties. Tetrameric $\mathrm{Hbs}$ from Antarctic fish are particularly well suited to this purpose, as they form a highly correlated system with peculiar structural and functional similarities and differences. These Hbs have $\mathrm{T}$ and $\mathrm{R}$ states very similar to those characterized in mammalian $\mathrm{Hb}$ [15-17]. However, they follow a peculiar oxidation pathway, that is characterized by a distinct behaviour of the $\alpha$ and $\beta$ chains $[18,19]$. Intermediate species with the $\alpha$ chain in the oxy, carbomonoxy or met state and the $\beta$ chain in the hexa-coordinate low-spin, $6 c \mathrm{LS}$, bis-histidyl form (hemichromes) [18-20] or in the penta-coordinate high-spin, $5 \mathrm{cHS}$, ferric form have been detected [20,21]. Interestingly, these mixed forms also present a quaternary organization somewhat intermediate between the classical $\mathrm{R}$ and $\mathrm{T}$ states (H state) [18-20]. Recently, these ferric states have been identified also in polar and polar-related fish Hbs by using a combination of Raman spectroscopy [22-24], EPR [20,21] and X-ray crystallography $[18-20,24]$.

The ferric forms of $\mathrm{Hb}$ are physiologically inert to further oxygenation, but several subsequent side reactions in the $\mathrm{Hb}$ auto-oxidation may interfere with or merge into other biochemical pathways, including the formation of a hemichrome. The relevance of this species spans from biomedical to physiological aspects [23]. For example, auto-oxidation is a serious problem that limits the storage time of acellular Hb-based blood substitutes [25]; also, hemichrome detection has been suggested as a valuable tool for tumour diagnosis [26]. The reaction of acetylphenylhydrazine (APH) with erythrocytes leads to hemichrome formation in healthy people and not in breast-cancer patients [26]. Some of the hypotheses on the functional role of 6cLS hemichrome were reviewed in [27]. The bis-histidyl complex can be involved in ligand binding [28,29], in the in vivo reduction of met-Hb, in Heinz body formation [27] and in nitric oxide scavenging.

Reduction of the ferric form of AFHb in solution produces a deoxy state [19]. Interestingly, in human $\mathrm{Hb}(\mathrm{HbA})$, thermally-generated hemichrome in trehalose glass [25], when thermally-reduced by glucose, converts to a ferrous deoxy [25] or to a hemochrome state [30], depending on the heating time.

In order to establish whether AFHb hemichromes encapsulated in the crystal phase can be converted into hemochromes, we chemically reduced the ferric HbTb crystals and followed the process by Raman microscopy. Similar to results obtained by short thermal reduction of trehalose-glass encapsulated ferric 
$\mathrm{HbA}$ and myoglobin [25], but unlike the long thermal reduction in trehalose glass of $\mathrm{HbA}$ and myoglobin [30], the crystal environment does not favour the formation of a ferrous 6cLS bis-histidyl adduct in $\mathrm{HbTb}$ [19]. As observed in solution, in the crystal state $\mathrm{HbTb}$ hemichrome converts into a 5cHS deoxy state. The transition from the $\mathrm{H}$ to the $\mathrm{T}$ quaternary structure produces severe surface cracks on protein crystals that cannot allow the X-ray diffraction data collection, hampering structural characterization of the process at the atomic level.

\section{Materials and methods}

\subsection{Purification and crystallization}

Purification and storage of $\mathrm{Hb}$ from T. bernacchii $(\mathrm{HbTb})$ was performed as previously reported [31]. $\mathrm{HbTb}$ was oxidized with $\mathrm{K}_{3} \mathrm{Fe}(\mathrm{CN})_{6}$ and the excess was removed by gel filtration on a Sephadex G-25 column. Crystallization of oxidized $\mathrm{HbTb}(\mathrm{ox}-\mathrm{HbTb})$ was carried out at $\mathrm{pH} 7.6$ and room temperature by liquid-diffusion, using a capillary, according to a previously described procedure [19]. These crystals correspond to the previously characterized $\alpha$ (aquo-met) $/ \beta$ (hemichrome) structure [19].

\subsection{Reduction of ox-HbTb crystals}

In order to obtain the structure of reduced ox- $\mathrm{HbTb}$, the crystals have been soaked for a few hours in a stabilizing solution containing $500 \mathrm{mM}$ dithionite. Unfortunately, the soaking procedure produced cracks on the crystals surface that prevented data collection. To reveal at least the first events in the reducing pathway of ox-HbTb, diffraction data on two crystals, soaked in a solution of dithionite for just few min (10 and $30 \mathrm{~min})$, have been collected at high resolution (1.60 and $1.76 \AA$, respectively) at the XRD1 beam-line of Elettra synchrotron. Data were collected at $100 \mathrm{~K}$ using glycerol as cryoprotectant and processed with the program suite HKL2000. The coordinates of the structure of the $\alpha$ (aquo-met) $/ \beta$ (hemichrome) form of HbTb (Protein Data Bank (PDB) code 2PEG), solved from crystals isomorphous to those here reported, were used as a starting model to refine the new structures. The refinement runs, performed using the program CNS [32], were followed by manual intervention using the molecular graphic program $\mathrm{O}$ [33] to correct minor errors in the position of few side chains. At convergence, the R-factor (R-free) values for the two structures were $0.211(0.234)$ and $0.195(0.222)$, respectively. Further diffraction and refinement statistics are listed in Table 1.

\subsection{Raman microscopy}

The reduction of ferric HbTb was also followed by resonance Raman (RR) microscopy. In the RR experiments carried out in solution the protein was kept in $50 \mathrm{mM}$ Tris- $\mathrm{HCl}$ (pH 7.5). The heme concentration was $0.5 \mathrm{mM}$. A confocal Raman microscope (Jasco, NRS-3100) was used to record Raman spectra. One of the three available lines (488 and $514 \mathrm{~nm}$ of an air-cooled $\mathrm{Ar}^{+}$laser, and $532 \mathrm{~nm}$ of a NdYAG laser) was injected into an integrated Olympus microscope and focused to a spot size of approximately $2 \mu \mathrm{m}$ by a $20 \times$ objective. A holographic notch filter was used to reject the excitation laser line. Raman scattering was dispersed through a monocromator (2400 grooves $/ \mathrm{mm}$ grating) and collected by a peltier-cooled $1024 \times 128$ pixel CCD photon detector (Andor DU401BVI). Typically, several $15 \mathrm{~min}$ solution spectra were recorded and averaged $\left(3-4 \mathrm{~cm}^{-1}\right.$ resolution) by a standard software routine. Frequency shifts were calibrated by using indene. Microscopy experiments were conducted on ferric $\mathrm{HbTb}$ 
Table 1

Diffraction and refinement statistics of short-time dithionite-soaked ox-HbTb crystals

\begin{tabular}{|c|c|c|}
\hline Data collection & $\begin{array}{c}\mathrm{ox}-\mathrm{HbTb} \\
10 \mathrm{~min} \text { in dithionite }\end{array}$ & $\begin{array}{c}\mathrm{ox}-\mathrm{HbTb} \\
30 \mathrm{~min} \text { in dithionite }\end{array}$ \\
\hline Space group & $\mathrm{C} 2$ & $\mathrm{C} 2$ \\
\hline \multicolumn{3}{|l|}{ Cell parameters } \\
\hline a $(\AA)$ & 87.158 & 87.405 \\
\hline $\mathrm{b}(\AA)$ & 87.762 & 87.687 \\
\hline c $(\AA)$ & 55.385 & 55.413 \\
\hline$\beta\left(^{\circ}\right)$ & 97.76 & 97.73 \\
\hline Resolution limits $(\AA)$ & $30.00-1.60$ & $30.0-1.76$ \\
\hline Highest resolution shell ( $\mathrm{A})$ & $1.65-1.60$ & $1.81-1.76$ \\
\hline No. of observations & 387,063 & 267,769 \\
\hline No. of unique reflections & 57,841 & 43,414 \\
\hline Completeness (\%) & $98.8(98.5)$ & $98.9(98.5)$ \\
\hline$I / \sigma(I)$ & $14.7(3.0)$ & $23.7(3.2)$ \\
\hline R-merge $(\%)$ & $7.4(24.1)$ & $5.5(39.0)$ \\
\hline Mosaicity & 0.53 & 0.53 \\
\hline \multicolumn{3}{|l|}{ Refinement results } \\
\hline Resolution limits $(\AA)$ & $20.00-1.60$ & $20.0-1.76$ \\
\hline Number of reflections used in the refinement & 51,243 & 38,218 \\
\hline No. of reflections in working set & 46,038 & 34,368 \\
\hline No. of reflections in test set & 5205 & 3850 \\
\hline R-working/R-free (\%) & $21.1 / 23.4$ & $19.5 / 22.2$ \\
\hline No. of protein atoms & 2199 & 2199 \\
\hline No. of water molecules & 319 & 324 \\
\hline \multicolumn{3}{|l|}{ RMSD from ideal values } \\
\hline Bond lengths $(\AA)$ & 0.009 & 0.010 \\
\hline Bond angles $\left({ }^{\circ}\right)$ & 1.21 & 1.21 \\
\hline
\end{tabular}

Note: Values in parentheses correspond to the highest resolution shells.

(ox-HbTb) crystals by previously reported procedures [34]. A complete data set on $\mathrm{HbTb}$ crystals was registered in $60 \mathrm{~s}$. Raman crystallography measurements on crystals mounted onto a goniometer head were performed by means of a modified Ventracon accessory.

\section{Results}

\subsection{X-ray diffraction data}

Crystals of ferric $\mathrm{HbTb}$ (ox-HbTb) were grown at $\mathrm{pH} 7.6$ according to a previous procedure [19, 20]. Single crystals of HbTb grew as large as $0.2 \times 0.2 \times 0.2 \mathrm{~mm}^{3}$. The crystal structure revealed an $\alpha$ (aquo-met) $/ \beta$ (hemichrome) state $[19,20]$. The quaternary structure of this form at $\mathrm{pH} 7.6$ is intermediate between $\mathrm{R}$ and $\mathrm{T}$ state. This new quaternary structure will be hereafter denoted as $\mathrm{H}$ state. The quaternary structure of the deoxy $\mathrm{HbTb}$ crystals from $\mathrm{pH} 6.0$ up to 8.4 showed a classical $\mathrm{T}$ state [16].

To characterize the reduction of the ox- $\mathrm{HbTb}$ structure at atomic level, crystals of ox- $\mathrm{HbTb}$ were soaked in a stabilizing solution containing $100 \mathrm{mM}$ dithionite, from a few min to a few hours. Diffrac- 
tion data on two HbTb crystals soaked in dithionite for 10 and 30 min were collected at 1.60 and $1.76 \AA$ resolution, respectively (Table 1). The structures obtained from these crystals reveal no significant differences from ox- $\mathrm{HbTb}$ (the root mean square deviation (RMSD) calculated superimposing the $\mathrm{C} \alpha$ atoms is only $0.13 \AA$ in both cases). The two structures are also very similar to each other (RMSD $<0.1 \AA$ ). The overall structure adopts the $\mathrm{H}$-state quaternary shape. The $\alpha$ heme is in $6 \mathrm{cHS}$ aquo-met/6cLS hydroxymet state, with the centre of the electron-density peak about $2.0 \AA$ away from the iron and about $4 \AA$ from the $N \varepsilon_{2}$ atom of distal His-59 $\alpha$. In the $\beta$ chains, a $6 c L S$ bis-histidyl coordination of the irons is observed. These data indicate that a few-minute dithionite soaking is not sufficient to significantly reduce the $\alpha$-heme iron of ox-HbTb crystals. Furthermore, no indication on the $\beta$-heme oxidation state is provided, since the 6cLS state for iron $\mathrm{Fe}^{2+}$ and $\mathrm{Fe}^{3+}$ are not expected to show relevant stereochemical differences. On the other hand, dithionite-soaking times of a few hours, caused severe surface cracks on the $\mathrm{HbTb}$ crystals that hamper data collection. This evidence indirectly suggests that the reduction of ox- $\mathrm{HbTb}$ involves a quaternary structure transition that is not compatible with the crystal packing of $\mathrm{HbTb}$.

\subsection{Raman microscopy}

Since the reduction of ox-HbTb cannot be properly investigated by X-ray crystallography studies, we followed this process using Raman microscopy. Ox-HbTb crystals, before and after reduction by dithionite, were characterized. The high-frequency region $\left(1300-1700 \mathrm{~cm}^{-1}\right)$ of the RR spectrum includes the porphyrin in-plane vibrational modes (that are sensitive to the electron density of the macrocycle), oxidation, coordination and spin state of the iron atom [35]. During most of the analysis, crystals were kept into a $1-\mu l$ drop of their mother liquor. High-frequency resonance Raman spectra of a solution and single crystal of ox-HbTb are reported in Fig. 1. The two spectra, except for an intensity factor, are indistinguishable. Therefore, the starting point of the reduction followed by Raman microscopy is the same as in solution. A tentative assignment of the Raman bands, based on previous studies [35], is reported in Table 2. The spectra of ox-HbTb crystals are characterized by a mixture of two ferric states, a $6 \mathrm{cHS}$ aquomet state $\left(\nu_{3}\right.$ and $\nu_{2}$ bands at 1476 and $\left.1561 \mathrm{~cm}^{-1}\right)$ and a $6 \mathrm{cLS}$ hemichrome state $\left(\nu_{3}\right.$ and $\nu_{2}$ bands at 1505 and $\left.1578 \mathrm{~cm}^{-1}\right)$. The X-ray crystal structure of ox- $\mathrm{HbTb}$ has assigned equal amounts to aquo-met and hemichrome, due to selective binding to the $\alpha$ and $\beta$ heme, respectively [19,20]. High-frequency Raman spectra of ferric $\mathrm{HbTb}$ single crystals have been collected using three excitation wavelengths (Fig. 2). The enhancement of Raman bands corresponding to the two ferric states is different when using the three wavelengths around the zone of the Q-bands, that should particularly enhance B-terms [36]. The 532-nm line particularly enhances the 6cLS hemichrome bands $\left(\nu_{3}=1506, \nu_{19}=1587\right.$ and $\nu_{10}=1640 \mathrm{~cm}^{-1}$ ), and it is expected to also enhance the bands of the 6cLS hemochrome state, namely the main target of this investigation. Therefore, we chose the $532 \mathrm{~nm}$ line to follow ox-HbTb reduction encapsulated into the crystal state.

Raman spectra of single crystals can be affected by the crystal orientation [37]. Therefore, prior to reduction of ferric $\mathrm{HbTb}$ crystals, we transferred a single crystal into a capillary ( $0.5 \mathrm{~mm}$-diameter) for X-ray diffraction, which was mounted onto a goniometer head for Raman crystallography analysis. The high-frequency spectra were recorded at different crystal orientations, showing that the crystal orientation does not affect the Raman spectrum of ox-HbTb (data not shown).

Reduction of the ferric $\mathrm{HbTb}$ crystals was initiated by adding a small volume of stabilizing solution containing dithionite to the drop of the crystal. Raman spectra at different dithionite additions are reported in Fig. 3. The quality of the spectra deteriorate with dithionite addition, but this is not surprising, 


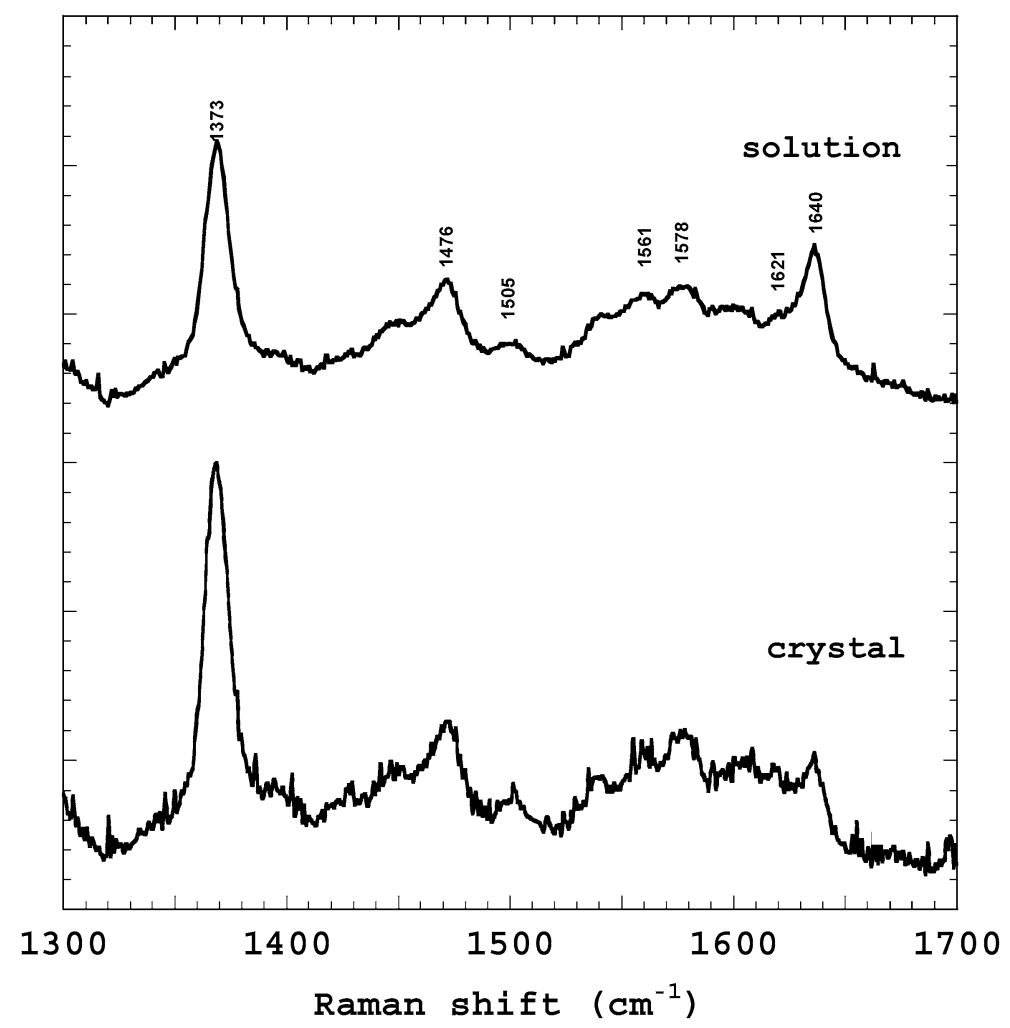

Fig. 1. RR spectra of ferric HbTb in crystal form (1-min acquisition) and solution (15-min acquisition) with 488-nm excitation line $(2.7 \mathrm{~mW}$ at the sample).

Table 2

Tentative assignment of the vibrational modes to ferric and reduced $\mathrm{HbTb}$ crystals

\begin{tabular}{lccc}
\hline Mode & $\begin{array}{c}\text { Ferric } \\
6 \mathrm{cLS}\end{array}$ & $\begin{array}{c}\text { Ferric } \\
6 \mathrm{cHS}\end{array}$ & $\begin{array}{c}\text { Ferrous } \\
5 \mathrm{cHS}\end{array}$ \\
\hline$\nu_{2}$ & 1578 & $1561^{*}$ & 1565 \\
$\nu_{3}$ & 1505 & 1476 & 1471 \\
$\nu_{4}$ & 1373 & 1371 & 1355 \\
$\nu_{10}$ & 1640 & 1610 & 1604 \\
$\nu_{19}$ & 1588 & $1561^{*}$ & 1555 \\
$\nu_{(\mathrm{C}=\mathrm{C})}$ & 1621 & 1621 & 1619 \\
& 1629 & 1629 & 1626 \\
$\mathrm{~d}_{\left(=\mathrm{CH}_{2}\right)}$ & 1435 & 1429 & 1426 \\
$\nu_{38}$ & 1543 & 1518 & 1524 \\
${ }^{*}$ Bands overlapping. & & &
\end{tabular}

since visual inspection in an optical microscope reveals wrinkles on the crystal surfaces. Upon dithionite addition, the oxidation marker $\nu_{4}$ and the core size band $\nu_{3}$ and $\nu_{10}$ show the appearance of the ferrous $5 \mathrm{cHS}$ deoxy state $\left(\nu_{4}=1355, \nu_{3}=1471, \nu_{11}=1548, \nu_{19}=1555\right.$ and $\left.\nu_{10}=1604 \mathrm{~cm}^{-1}\right)$. Concomitantly, the ferric species $\left(\nu_{4}=1373 \mathrm{~cm}^{-1}\right)$, and more specifically the $6 \mathrm{cLS}$ ferric form $\left(\nu_{3}=1505\right.$ and $\left.\nu_{10}=1640 \mathrm{~cm}^{-1}\right)$, tends to disappear. The putative $6 \mathrm{cLS}$ hemochrome state should appear at $\nu_{4}=1361$ 


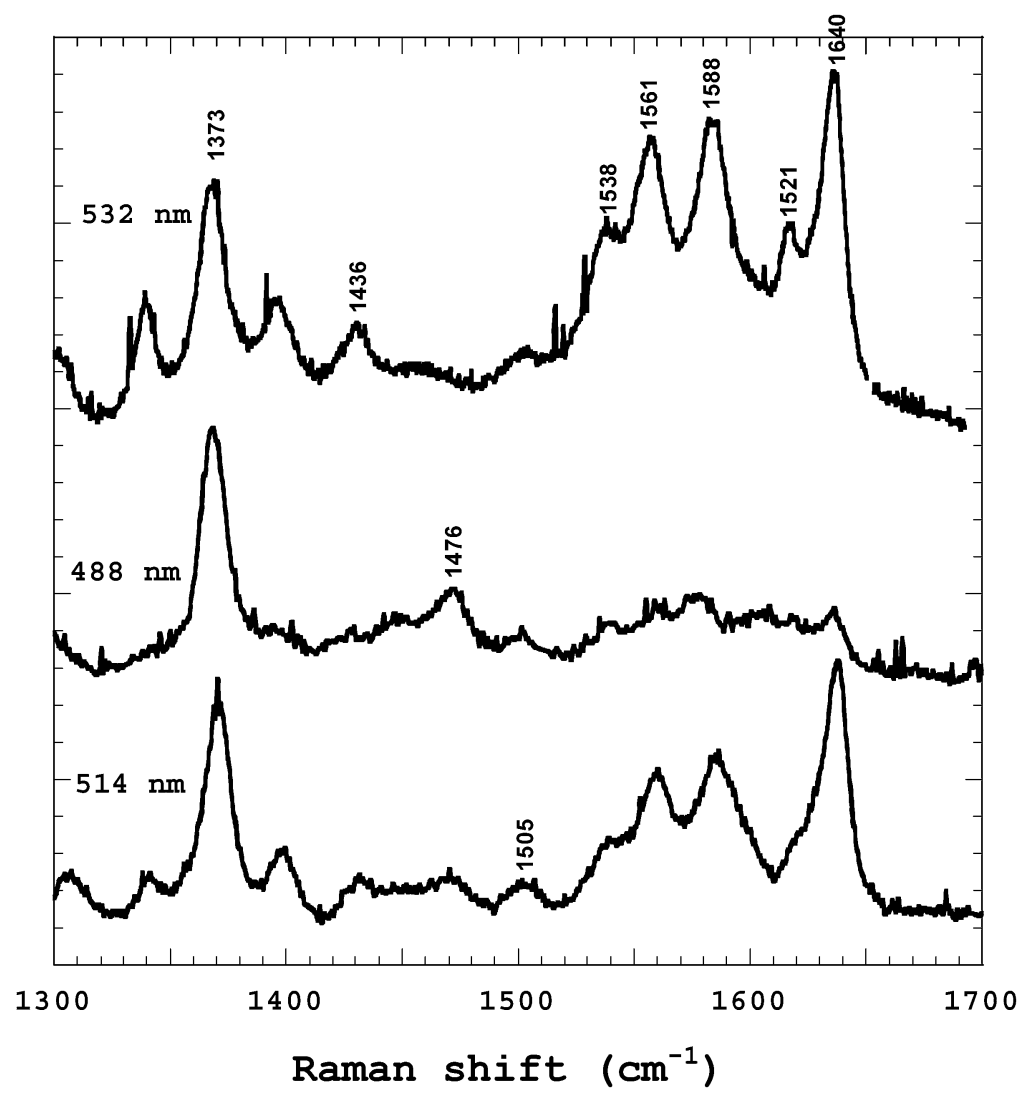

Fig. 2. RR spectra of ferric HbTb in crystal form (1-min acquisition) with 488-nm (2.7 mW at the sample), $514-\mathrm{nm}$ (3 mW at the sample) and 532-nm (0.7 $\mathrm{mW}$ at the sample) excitation lines.

and $\nu_{3}=1493 \mathrm{~cm}^{-1}[38,39]$. On the contrary, the final spectrum in Fig. 4 contains bands associated with non-totally symmetric skeletal modes $\left(\nu_{10}, \nu_{19}\right.$ and $\left.\nu_{11}\right)$, and it matches well the spectrum obtained at $532 \mathrm{~nm}$ for deoxy $\mathrm{HbA}$ [40]. Indeed, this reference spectrum contains a broad overlapped band $\left(\nu_{19}\right.$ plus $\left.\nu_{11}\right)$ with a maximum at $1550 \mathrm{~cm}^{-1}$, and another band $\nu_{10}$ at $1604 \mathrm{~cm}^{-1}$ [40]. Though the final spectrum is deteriorated by partial crystal dissolution, it mostly corresponds to that of a deoxy state; if present, only a minor amount of a ferrous 6cLS species is formed. The Raman microscopy data on crystals are in perfect agreement with the spectroscopic data in solution [19].

\section{Discussion}

In principle, when reduced by dithionite, the $\alpha$ (aquo-met) $/ \beta$ (hemichrome) crystals may be converted to a mixture of high-spin penta-coordinate (5cHS) deoxy state and low-spin hexa-coordinate (6cLS) hemochrome state. In fact, the crystal phase could hamper the scissor-like motion that helices EF undergo to disrupt the endogenous bis-histidyl coordination.

We have characterized the reduction of $\mathrm{HbTb}$ hemichrome in a crystal phase. Unfortunately, X-ray diffraction experiments cannot be performed on reduced ox- $\mathrm{HbTb}$ crystals, given that the addition of dithionite produces severe crystal damage that hamper data collection. However, experiments on these 


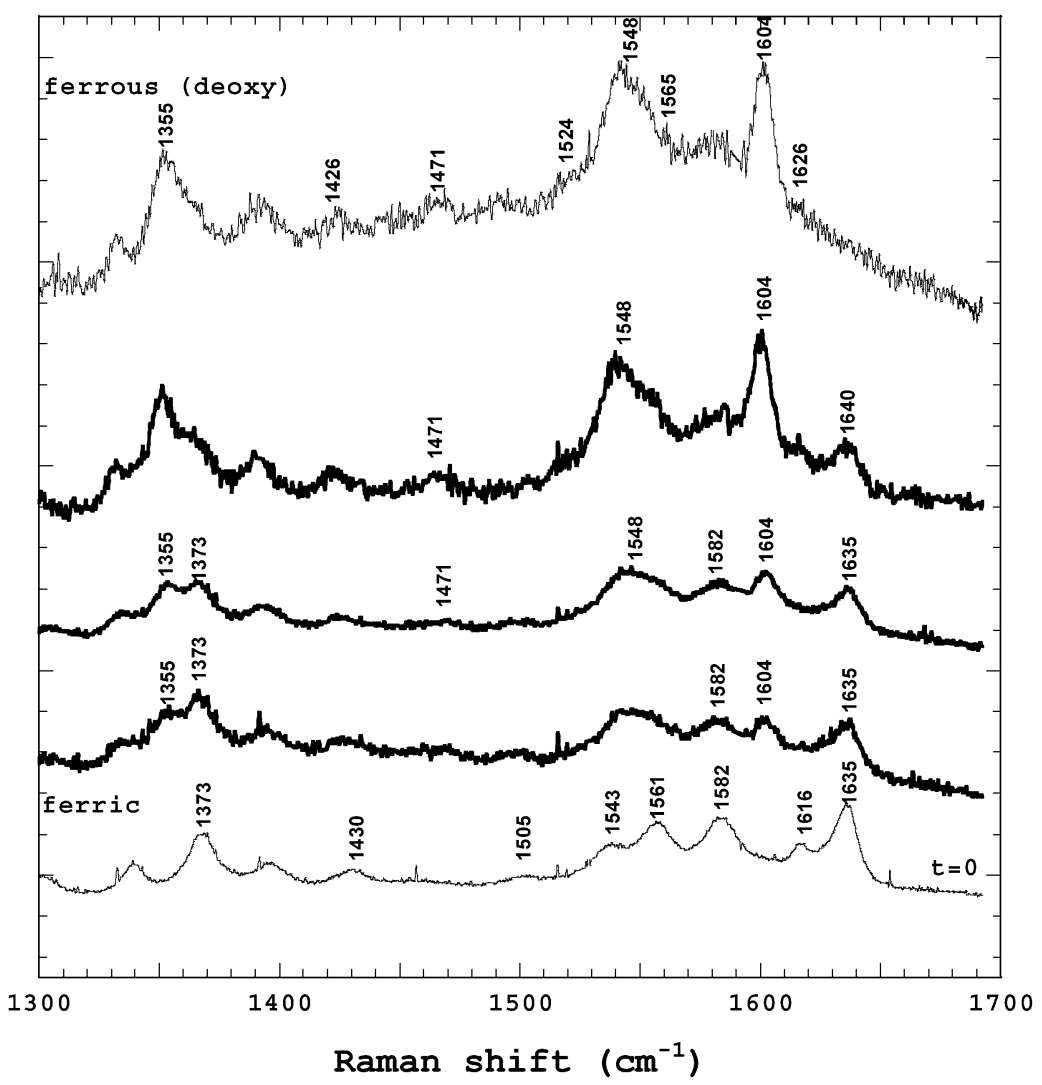

Fig. 3. RR spectra of HbTb crystal (1-min acquisition) with $532 \mathrm{~nm}(0.7 \mathrm{~mW}$ at the sample) excitation line. Ferric form (bottom spectrum), ferrous form (top spectrum) and partially reduced forms (intermediate spectra) at different dithionite additions.

crystals suggest that the reduction process of ox-HbTb is a cooperative phenomenon, involving a conformational variation that cannot be accommodated into the protein crystal. These indirect indications are in full agreement with the Raman microscopy data, showing that the 6cLS bis-histidyl coordination is loosened in favour of a 5cHS ferrous deoxy state, with no evidence of ferrous 6sLS bis-histidyl state. Upon reduction, the hemichrome converts to a deoxy ferrous state; the quaternary structure of ox- $\mathrm{HbTb}$ must change from the $\mathrm{H}[20]$ to the $\mathrm{T}$ state [16]. This large conformational variation produces increased disorder in the protein crystal that decreases its diffraction power. The conversion of hemichrome in $\mathrm{HbTb}$ to the deoxy state is also in agreement with the data on reduction of ferric $\mathrm{HbTb}$ in solution [19]. Altogether these analyses suggest that in Antarctic fish Hbs (AFHb), the bis-histidyl adduct is typical only of the ferric state (hemichrome). In several Hbs, both hemochrome and hemichrome occur, and in other systems only hemochrome has been observed [23]. Despite the structural similarities in the local stereochemistry of the two compounds, and the restrictions caused by the crystal packing, the hemochrome does not replace efficiently the hemichrome species. The interpretation of such a result requires a quantum-mechanical approach that is under way in our laboratory.

The results of this work indicate that, even though bis-histidyl adducts have a functional significance in AFHbs, this function refers to a stable ferric state, or to a transient, though never detected, ferrous species. 


\section{Acknowledgements}

This work was financially supported by PNRA (Italian National Programme for Antarctic Research). We thank the ELETTRA Synchrotron (Trieste, Italy) for beam-time and the staff of the beam-line XRD1 for assistance during data collection. Giosuè Sorrentino and Maurizio Amendola are acknowledged for their technical assistance.

\section{References}

[1] W.A. Eaton, E.R. Henry, J. Hofrichter and A. Mozzarelli, Is cooperative oxygen binding by hemoglobin really understood?, Nat. Struct. Biol. 6(4) (1999), 351-358.

[2] A. Bellelli, M. Brunori, A.E. Miele, G. Panetta and B. Vallone, The allosteric properties of hemoglobin: insights from natural and site directed mutants, Curr. Protein Pept. Sci. 7(1) (2006), 17-45.

[3] T. Yonetani and A. Tsuneshige, The global allostery model of hemoglobin: an allosteric mechanism involving homotropic and heterotropic interactions, C. R. Biol. 326(6) (2003), 523-532.

[4] D. Barrick, N.T. Ho, V. Simplaceanu, F.W. Dahlquist and C. Ho, A test of the role of the proximal histidines in the Perutz model for cooperativity in haemoglobin, Nat. Struct. Biol. 4(1) (1997), 78-83.

[5] M.F. Perutz, A.J. Wilkinson, M. Paoli and G.G. Dodson, The stereochemical mechanism of the cooperative effects in hemoglobin revisited, Annu. Rev. Biophys. Biomol. Struct. 27 (1998), 1-34.

[6] M.F. Perutz, Stereochemistry of cooperative effects in haemoglobin, Nature 228(273) (1970), 726-739.

[7] M.K. Safo and D.J. Abraham, The enigma of the liganded hemoglobin end state: a novel quaternary structure of human carbonmonoxy hemoglobin, Biochemistry 44(23) (2005), 8347-8359.

[8] J.R. Tame, What is the true structure of liganded haemoglobin?, Trends Biochem. Sci. 24(10) (1999), 372-377.

[9] M.M. Silva, P.H. Rogers and A. Arnone, A third quaternary structure of human hemoglobin A at 1.7-A resolution, J. Biol. Chem. 267(24) (1992), 17248-17256.

[10] R. Srinivasan and G.D. Rose, The T-to-R transformation in hemoglobin: a reevaluation, Proc. Natl. Acad. Sci. USA 91(23) (1994), 11113-11117.

[11] A.J. Sutherland-Smith, H.M. Baker, O.M. Hofmann, T. Brittain and E.N. Baker, Crystal structure of a human embryonic haemoglobin: the carbonmonoxy form of gower II (alpha2 epsilon2) haemoglobin at 2.9 A resolution, J. Mol. Biol. 280(3) (1998), 475-484.

[12] M.K. Safo and D.J. Abraham, The X-ray structure determination of bovine carbonmonoxy hemoglobin at 2.1 A resolution and its relationship to the quaternary structures of other hemoglobin crystal forms, Protein Sci. 10(6) (2001), 1091-1099.

[13] T.C. Mueser, P.H. Rogers and A. Arnone, Interface sliding as illustrated by the multiple quaternary structures of liganded hemoglobin, Biochemistry 39(50) (2000), 15353-15364.

[14] M.A. Schumacher, E.E. Zheleznova, K.S. Poundstone, R. Kluger, R.T. Jones and R.G. Brennan, Allosteric intermediates indicate R2 is the liganded hemoglobin end state, Proc. Natl. Acad. Sci. USA 94(15) (1997), 7841-7844.

[15] L. Mazzarella, R. D'Avino, G. di Prisco et al., Crystal structure of Trematomus newnesi hemoglobin re-opens the Root effect question, J. Mol. Biol. 287(5) (1999), 897-906.

[16] L. Mazzarella, A. Vergara, L. Vitagliano et al., High resolution crystal structure of deoxy hemoglobin from Trematomus bernacchii at different $\mathrm{pH}$ values: the role of histidine residues in modulating the strength of the Root effect, Proteins Struct. Funct. Bioinf. 65 (2006), 490-498.

[17] L. Mazzarella, G. Bonomi, M.C. Lubrano et al., Minimal structural requirement of Root effect: crystal structure of the cathodic hemoglobin isolated from Trematomus newnesi, Proteins Struct. Funct. Bioinf. 62(2) (2006), 316-321.

[18] A. Riccio, L. Vitagliano, G. di Prisco, A. Zagari and L. Mazzarella, The crystal structure of a tetrameric hemoglobin in a partial hemichrome state, Proc. Natl. Acad. Sci. USA 99(15) (2002), 9801-9806.

[19] L. Vitagliano, G. Bonomi, A. Riccio, G. di Prisco, G. Smulevich and L. Mazzarella, The oxidation process of Antarctic fish hemoglobins, Eur. J. Biochem. 271(9) (2004), 1651-1659.

[20] A. Vergara, M. Franzese, A. Merlino et al., Structural characterization of ferric hemoglobins from three Antarctic fish species of the suborder Notothenioidei, Biophys. J. 93 (2007), 2822-2829.

[21] D. Giordano, A. Vergara, H.C. Lee et al., Hemoglobin structure/function and globin-gene evolution in the Arctic fish Liparis tunicatus, Gene 406(1,2) (2007), 48-58.

[22] C. Verde, B.D. Howes, M.C. de Rosa et al., Structure and function of the Gondwanian hemoglobin of Pseudaphritis urvillii, a primitive notothenioid fish of temperate latitudes, Prot. Sci. 13(10) (2004), 2766-2781.

[23] A. Vergara, L. Vitagliano, G. di Prisco, C. Verde and L. Mazzarella, Spectroscopic and crystallographic characterization of hemichromes in tetrameric hemoglobins, Meth. Enz. 436A (2008), 421-440. 
[24] L. Vitagliano, A. Vergara, G. Bonomi et al., Spectroscopic and crystallographic analysis of a tetrameric hemoglobin oxidation pathway reveals features of an intermediate R/T state, (2007), submitted.

[25] A. Ray, B.A. Friedman and J.M. Friedman, Trehalose glass-facilitated thermal reduction of metmyoglobin and methemoglobin, J. Am. Chem. Soc. 124(25) (2002), 7270-7271.

[26] S. Croci, G. Pedrazzi, G. Passeri, P. Piccolo and I. Ortalli, Acetylphenylhydrazine induced haemoglobin oxidation in erythrocytes studied by Mossbauer spectroscopy, Biochim. Biophys. Acta 1568(1) (2001), 99-104.

[27] J.M. Rifkind, O. Abugo, A. Levy and J.M. Heim, Detection, formation, and relevance of hemichrome and hemochrome, Meth. Enz. 231 (1994), 449-480.

[28] D. de Sanctis, A. Pesce, M. Nardini, M. Bolognesi, A. Bocedi and P. Ascenzi, Structure-function relationships in the growing hexa-coordinate hemoglobin sub-family, IUBMB Life 56(11/12) (2004), 643-651.

[29] A. Pesce, D. De Sanctis, M. Nardini et al., Reversible hexa- to penta-coordination of the heme Fe atom modulates ligand binding properties of neuroglobin and cytoglobin, IUBMB Life 56(11/12) (2004), 657-664.

[30] M. Navati and J.M. Friedman, Sugar-derived glasses support thermal and photo-initiated electron transfer processes over macroscopic distances, J. Biol. Chem. 281 (2006), 36021-36028.

[31] L. Camardella, C. Caruso, R. D'Avino et al., Hemoglobin of the Antarctic fish Pagothenia bernacchii. Amino acid sequence, oxygen equilibria and crystal structure of its carbonmonoxy derivative, J. Mol. Biol. 224 (1992), 449-460.

[32] A.T. Brunger, P.D. Adams, G.M. Clore et al., Crystallography \& NMR system: A new software suite for macromolecular structure determination, Acta Crystallogr. D - Biol. Crystallogr. 54 (1998), 905-921.

[33] T.A. Jones, J.Y. Zou, S.W. Cowan and M. Kjedgaard, Improved methods for binding protein models in electron density maps and the location of errors in these models, Acta Crystallogr. D - Biol. Crystallogr. 56 (1991), 714-721.

[34] P.R. Carey, Raman crystallography and other biochemical applications of Raman microscopy, Annu. Rev. Phys. Chem. 57 (2006), 527-554

[35] S. Choi, T.G. Spiro, K.C. Langry, K.M. Smith, D.L. Budd and G.N. La Mar, Structural correlation and vinyl influences in resonance Raman spectra of protoheme complexes and protein, J. Am. Chem. Soc. 104 (1982), 4345-4351.

[36] T.G. Spiro, Iron Porphyrins, Vol. II, A. Lever and H. Gray, eds, Addison-Wesley, Reading, MA, 1983.

[37] G. Smulevich and T.G. Spiro, Single-crystal micro-Raman spectroscopy, Meth. Enzymol. 226 (1993), 397-408.

[38] T.K. Das, H.C. Lee, S.M.G. Duff et al., The heme environment in barley hemoglobin, J. Biol. Chem. 274(7) (1999), $4207-4212$

[39] M. Couture, T.K. Das, H.C. Lee et al., Chlamydomonas chloroplast ferrous hemoglobin. Heme pocket structure and reactions with ligands, J. Biol. Chem. 274(11) (1999), 6898-6910.

[40] S. Dasgupta and T.G. Spiro, Resonance Raman charcaterization of the 7-ns photoproduct of (carbomonoxy)hemoglobin: implication for hemoglobin dynamics, Biochemistry 25 (1986), 5941-5948. 


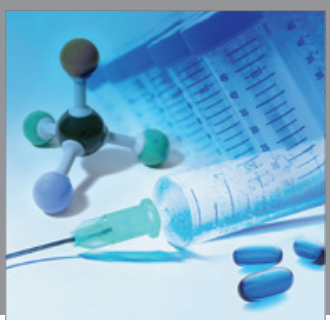

International Journal of

Medicinal Chemistry

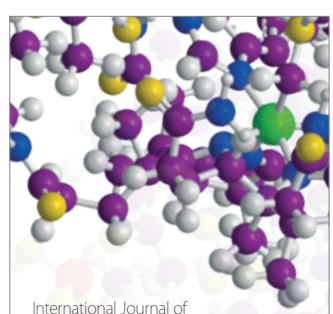

Carbohydrate Chemistry

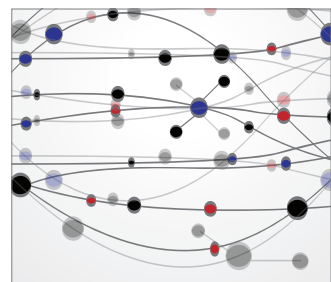

The Scientific World Journal
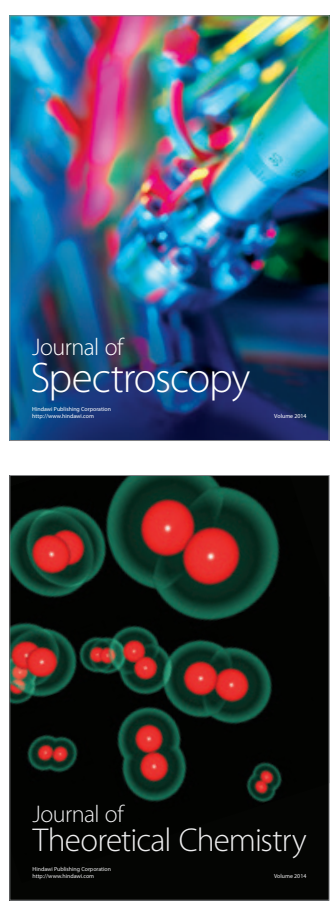
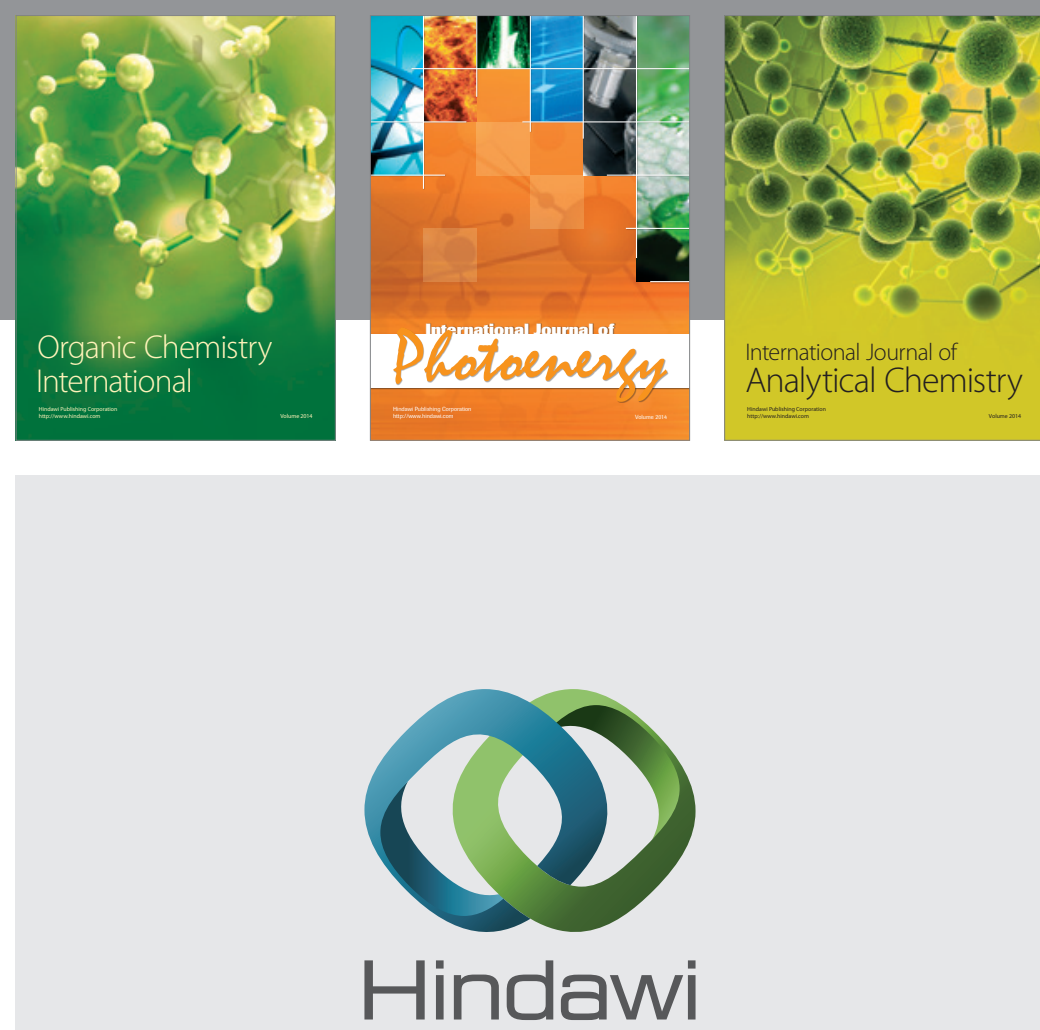

Submit your manuscripts at

http://www.hindawi.com
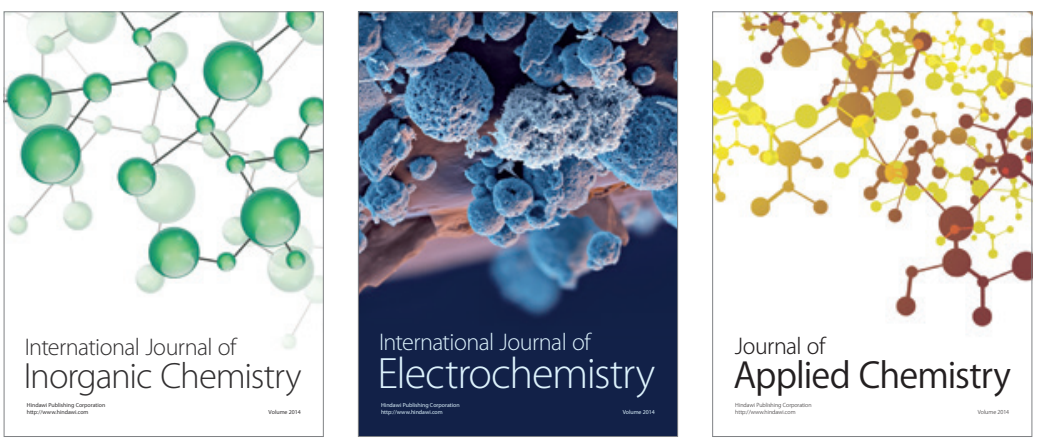

Journal of

Applied Chemistry
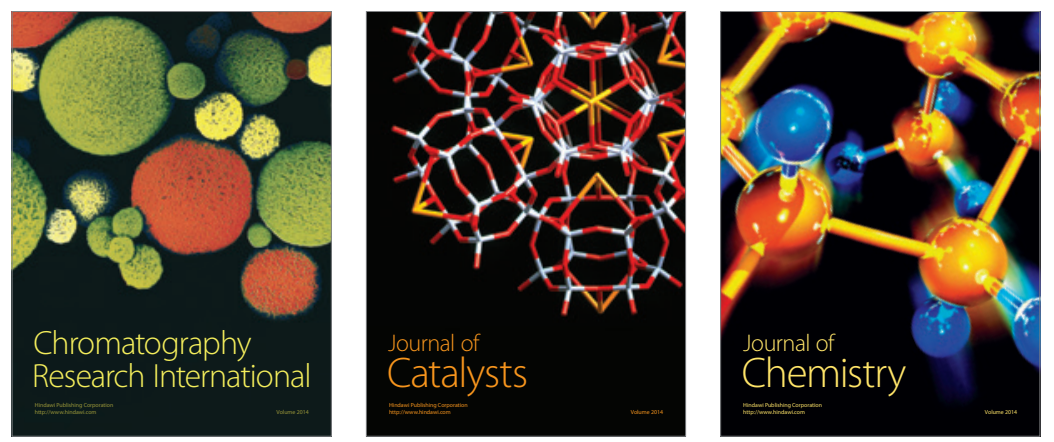
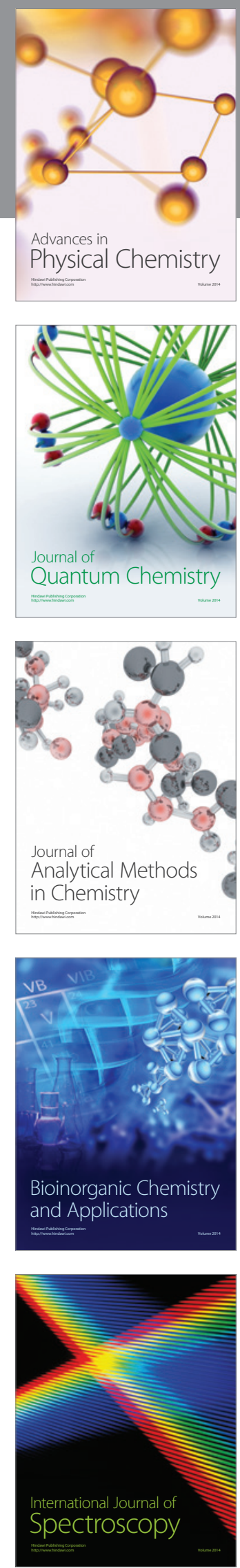